\title{
Lesiones oncológicas intracraneales de apariencia quística
}

\section{Oncologic Intracranial Lesions with Cystic Appearance}

\author{
Pablo Sartori ${ }^{1}$ Nicolás Sgarbi ${ }^{2}$ \\ ${ }^{1}$ Diagnóstico Mediter, Ciudad Autónoma de Buenos Aires, Buenos \\ Aires, Argentina \\ ${ }^{2}$ Departamento Clínico de Radiología, Hospital de Clínicas de \\ Montevideo, Montevideo, Uruguay
}

\author{
Address for correspondence Pablo Sartori, Av. Avellaneda 551, \\ Ciudad Autónoma de Buenos Aires, Argentina \\ (e-mail: pablomsar@yahoo.com.ar).
}

Rev Argent Radiol 2021;85:11-20.

\section{Resumen \\ Palabras Clave \\ - encéfalo \\ - quistes \\ - neoplasias \\ - tomografía \\ - imágenes por resonancia magnética}

La presencia de lesiones quísticas de etiología oncológica en el encéfalo es cada vez más frecuente. El rol del especialista en imágenes es describir sus características, morfología y comportamiento con el contraste endovenoso, para llegar a realizar un diagnóstico presuntivo y enumerar sus diagnósticos diferenciales, y así orientar al médico tratante. Haremos una revisión de las lesiones quísticas de etiología oncológica de presentación frecuente en el encéfalo.

The presence of an oncologic cystic formation in the brain is frequent. The imaging specialist's role is to describe its features, morphology, and image enhancement characteristics with endovenous contrast, to make a presumptive diagnosis, and enumerated differential diagnoses and thus orientate the practitioner. We will analyze the most common expansive cystic formations.

\section{Introducción}

Una imagen quística puede definirse como una cavidad de contenido líquido o fluido semisólido revestida por un epitelio. ${ }^{1-3}$
Las lesiones expansivas pueden ser completamente quísticas o presentar áreas cavitadas y se subclasifican según su localización en intra o extraaxiales. received January 20, 2020 accepted July 20, 2020 published online December 22, 2020
DOI https://doi.org/ $10.1055 / \mathrm{s}-0040-1718460$. ISSN 1852-9992.

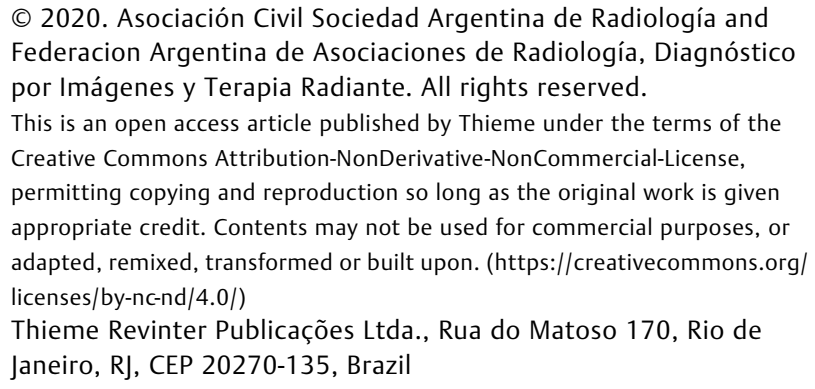

(C) 2020. Asociación Civil Sociedad Argentina de Radiología and Federacion Argentina de Asociaciones de Radiología, Diagnóstico por Imágenes y Terapia Radiante. All rights reserved. This is an open access article published by Thieme under the terms of the Creative Commons Attribution-NonDerivative-NonCommercial-License, permitting copying and reproduction so long as the original work is given appropriate credit. Contents may not be used for commercial purposes, or adapted, remixed, transformed or built upon. (https://creativecommons.org/ licenses/by-nc-nd/4.0/) Thieme Revinter Publicações Ltda., Rua do Matoso 170, Rio de Janeiro, RJ, CEP 20270-135, Brazil 
Tabla 1 Lesiones neoplásicas intracraneales de apariencia quística

\begin{tabular}{|c|c|}
\hline Localización & Ejemplos \\
\hline \multirow[t]{10}{*}{ Intra-axial } & ${ }^{*}$ Astrocitoma pilocítico \\
\hline & * Hemangioblastoma \\
\hline & ${ }^{*}$ Neurocitoma central \\
\hline & * Ependimoma \\
\hline & ${ }^{*}$ Meduloblastoma \\
\hline & * Ganglioglioma \\
\hline & * Xantoastrocitoma pleomórfico \\
\hline & * Tumor disembrioplásico embrionario \\
\hline & * Glioblastoma multiforme \\
\hline & * Metástasis \\
\hline \multirow[t]{4}{*}{ Extra-axial } & * Meningioma \\
\hline & *Schwanomma \\
\hline & ${ }^{*}$ Macroadenoma hipofisario \\
\hline & ${ }^{*}$ Craneofaringioma \\
\hline
\end{tabular}

Fuente: Referencias bibliográficas 4, 5.

Las lesiones expansivas intracraneales con componente quístico más frecuentes se detallan en la - Tabla 1.
El propósito de este trabajo es ilustrar el amplio espectro de lesiones quísticas intracraneales de origen neoplásico y describir sus características morfológicas.

\section{Tumores intra-axiales ${ }^{4,5}$}

Los diagnósticos diferenciales se mencionan en la - Tabla 2:

1) Astrocitoma pilocítico

Es el tumor de estirpe glial más frecuente en niños y adultos jóvenes, suele localizarse próximo a la línea media, $2 / 3$ se encuentran a nivel del cerebelo, en el quiasma óptico, alrededor del III ventrículo y en la región hipotálamohipofisaria; cuando se presenta en adultos, la mitad de ellos es supratentorial. ${ }^{4,6,7}$

Se ha reportado asociación entre los astrocitomas cerebelosos y la neurofibromatosis tipo I, la enfermedad de Ollier y los síndromes de Turcot y PHACE. ${ }^{4,6-8}$

Los signos y síntomas dependen de su localización, siendo las manifestaciones más frecuentes las derivadas de la hipertensión endocraneal. ${ }^{6}$

Se presentan como una masa redondeada-ovoidea sólido/ quística bien delimitada, con nódulo mural sólido $\mathrm{y}$ ocasionalmente centro necrótico. ${ }^{6,7}$

Existe una variante más agresiva denominada pilomixoide. ${ }^{6}$

En TC se aprecian como masas expansivas iso-hipodensas sólido/quísticas, las calcificaciones son infrecuentes, realzando el componente sólido, tras la administración del contraste endovenoso (e.v. $)^{4,6,7}$

Tabla 2 Diagnósticos diferenciales de lesiones neoplásicas intracraneales de apariencia quística

\begin{tabular}{|l|l|}
\hline Tumores con componente quístico & Diagnósticos diferenciales \\
\hline Astrocitoma pilocítico & $\begin{array}{l}\text { Ependimoma y meduloblastoma (niños en la fosa posterior) } \\
\text { Metástasis o hemangioblastoma (adultos) }\end{array}$ \\
\hline Hemangioblastoma & Astrocitoma pilocítico \\
\hline Neurocitoma central & $\begin{array}{l}\text { Oligodendroglioma, astrocitoma pilocítico, astrocitoma subepen- } \\
\text { dimario de células gigantes, ependimoma, meningiomas }\end{array}$ \\
\hline Craniofaringioma & $\begin{array}{l}\text { Adenoma hipofisario, glioma hipotalámico o de la vía óptica, quiste } \\
\text { de la bolsa de Rathke, quiste epidermoide, aneurismas trombosados }\end{array}$ \\
\hline Ependimoma & $\begin{array}{l}\text { Supratentoriales intraparenquimatosos: astrocitoma pilocítico, } \\
\text { xantoastrocitoma pleomórfico, tumor neuroectodérmico primitivo, } \\
\text { ganglioglioma/citoma y oligodendroglioma }\end{array}$ \\
\hline Meduloblastoma & $\begin{array}{l}\text { Astrocitoma pilocítico, tumor teratoide rabdoide atípico y } \\
\text { ependimoma }\end{array}$ \\
\hline Ganglioglioma & Ependimoma \\
\hline Xantoastrocitoma pleomórfico & Meningioma, glioblastoma, DNET, oligodendroglioma, metástasis \\
\hline Tumor neuroepitelial disembrioplásico (DNET) & Gangliocitoma, xantoastrocitoma pilocítico \\
\hline Glioblastoma & Metástasis, abscesos \\
\hline Metástasis & Abscesos, glioblastoma, astrocitoma, xantoastrocitoma pleomorfo, \\
\hline Meningiomas & Schwanommas \\
\hline Schwanommas & Meningiomas \\
\hline Macroadenoma hipofisario/craniofaringioma & Craniofaringioma/macroadenoma hipofisario \\
\hline
\end{tabular}

Fuente: Referencias bibliográficas 6, 8-10, 14-17, 19, 22, 28, 31, 32. 

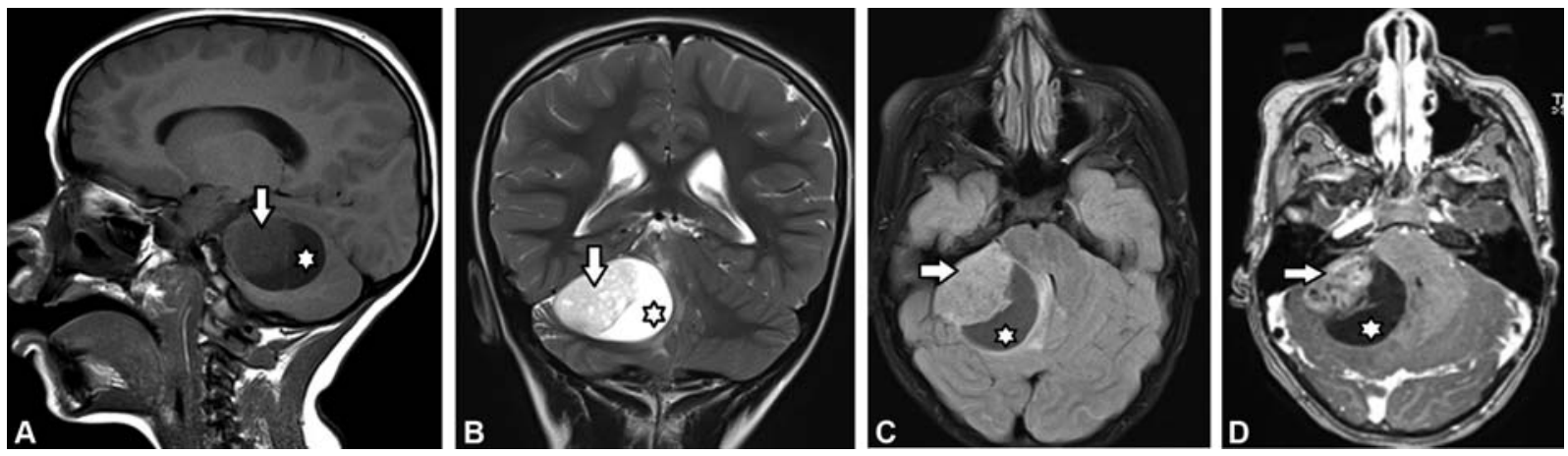

Fig. 1 RM de cerebro. Cortes: sagital ponderado en T1 (A), coronal ponderado en T2 (B), axial FLAIR (C) y axial ponderado en T1 con gadolinio (d). Paciente masculino de 7 años con cefaleas y mareos, apreciándose en el hemisfério cerebeloso derecho una lesión expansiva a predominio quística (estrella) con nódulo mural sólido excéntrico (flechas). Genera efecto de masa desplazando la línea media hacia la izquierda, rodeada de leve edema vasogénico. La porción sólida realza irregularmente tras la administración de gadolinio. Considerando la edad y forma de presentación, es compatible con astrocitoma pilocítico (confirmado mediante biopsia).

En RM, la porción quística tiene la misma señal que el líquido cefalorraquídeo (LCR) en secuencias ponderadas en T y T2, hiperintenso en FLAIR y se asocia a un nódulo mural isointenso en secuencias ponderadas en $\mathrm{T} 1$ e hiperintenso en T2 y FLAIR, que generalmente se realza tras la administración de gadolinio (-Fig. 1) ${ }^{4-7}$

Tanto el quiste como la porción sólida pueden realzar con gadolinio. 6,7

\section{2) Hemangioblastoma}

Es un tumor vascular infrecuente, generalmente asociado a pacientes con enfermedad de Von Hippel Lindau, aunque puede ser de presentación esporádica. ${ }^{4,8}$

Se presenta en jóvenes a nivel del cerebelo o menos frecuentemente en la médula espinal. ${ }^{4,8}$

En TC se observan como masas sólido-quísticas en la fosa posterior realzando la porción sólida con contraste e.v. ${ }^{8}$

En RM aparecen iso/hipointensos en secuencias ponderadas en T1 e hiperintensos en T2. Poseen un nódulo mural sólido que realza intensamente (-Fig. 2 ). ${ }^{8}$

En secuencias ponderadas en T2 muestran imágenes con vacío de señal por su alta vascularización. ${ }^{8}$ No suelen calcificar y la presencia de imágenes espontáneamente hiperintensas valoradas en secuencias ponderadas en T1 implica signos de sangrado reciente. ${ }^{8}$

La angiografía digital es una herramienta diagnóstica que puede ser considerada, dada su rica vascularización.

3) Neurocitoma central
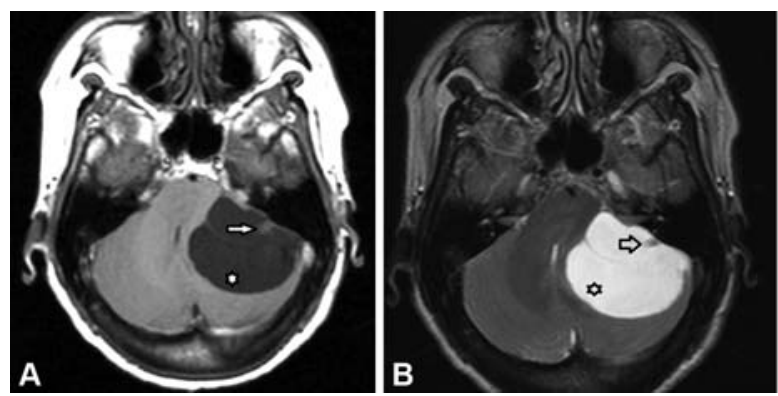

Es un tumor benigno antiguamente conocido como "ependimoma del foramen de Monro" u "oligodendroglioma intraventricular."9

Habitualmente intraventricular, situado en los ventrículos laterales próximo al agujero de Monro, aunque puede tener otras localizaciones (núcleos grises, IV ventrículo, intramedulares, etc.), suele afectar a adultos jóvenes y tener un pronóstico favorable. ${ }^{4,9-11}$

Los neurocitomas centrales se presentan con síntomas de hipertensión endocraneana por hidrocefalia obstructiva. ${ }^{9-11}$

Se aprecian como lesiones heterogéneas con componente sólido/quístico, de límites demarcados y contornos lobulados, en contacto con el septum pellucidum. ${ }^{4,9-11}$

Son heterogéneos en TC; en RM muestran apariencia "burbujeante" debido a la presencia de quistes. Son isointensos en secuencias ponderadas en T1, isohiperintensos en $\mathrm{T} 2$, presentando calcificaciones asociadas en el $50 \%$ de los casos (-Fig. 3). ${ }^{4,9,11}$

Realzan irregularmente y pueden sangrar. ${ }^{9-11}$

4) Ependimoma

De localización supra (40\%) o infratentorial (60\%). Los primeros pueden afectar al parénquima encefálico o a las cavidades ventriculares, siendo más frecuentes en jóvenes. Los que afectan la fosa posterior se presentan en niños. ${ }^{12,13}$

La sintomatología varía y depende de la localización (supra/infratentorial), pudiendo ocasionar síntomas por hipertensión intracraneal (frecuentemente los

Fig. 2 RM de cerebro. Cortes: axial ponderado en T1 (A), axial ponderado en T2 (B), axial (C) y coronal ponderado en T1 (D) con gadolinio. Se aprecia, en el hemisferio cerebeloso izquierdo (estrella), una lesión expansiva quística con septos en su interior con un nódulo mural periférico. La porción sólida realza intensamente tras la administración de gadolinio (flechas). Por anatomía patológica se confirmó un hemangioblastoma. 

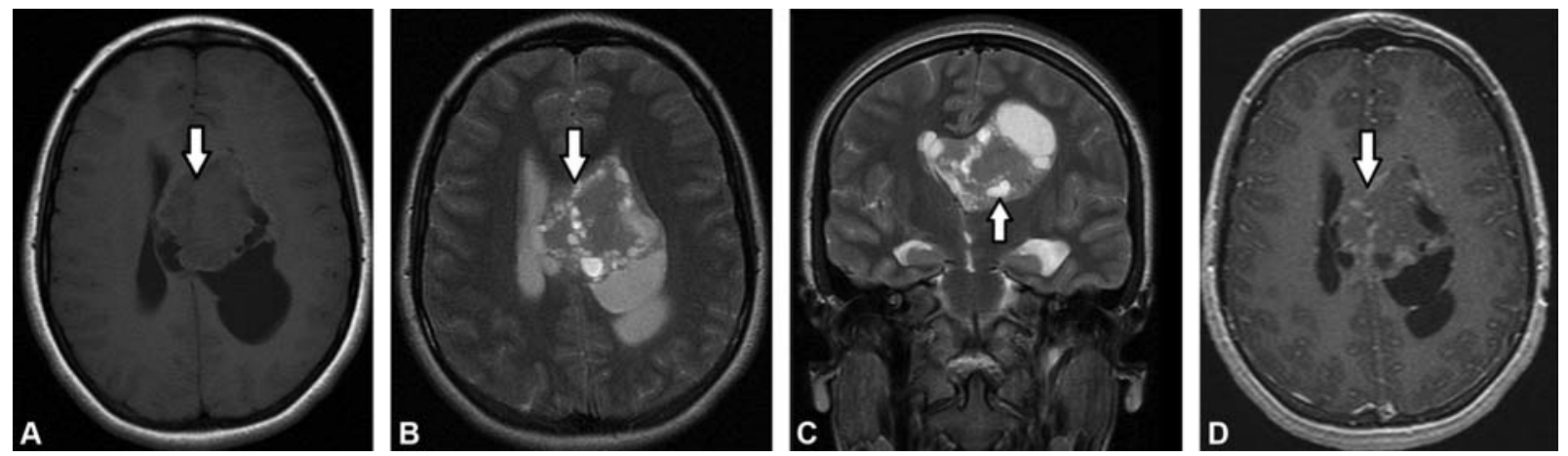

Fig. 3 RM cerebral. Cortes: axial ponderado en T1 (A), ponderado en T2 (B), coronal T2 (C) y axial ponderado en T1 con gadolinio (D). En la vecindad del agujero de Monro izquierdo se origina una lesión expansiva sólida, conteniendo múltiples imágenes quísticas (flechas). Genera desplazamiento de la línea media e hidrocefalia obstructiva supratentorial izquierda. Realza en forma heterogénea con gadolinio. Mediante biopsia se diagnosticó un neurocitoma central.

infratentoriales, debido a su localización intraventricular). Los pacientes con ependimomas supratentoriales pueden tener cefaleas, convulsiones o déficits neurológicos. ${ }^{12,13}$

Se presentan como masas con áreas quísticas y calcificaciones groseras y en ocasiones sangran. ${ }^{12}$

Los supratentoriales son de mayor tamaño (por su localización generalmente intraparenquimatosa) y tienden a tener componente quístico, mientras que los infratentoriales son frecuentemente intraventriculares, por lo que su tamaño es menor. ${ }^{13}$

En TC las áreas sólidas son iso/hipodensas y en RM son iso/ hipointensas en seuencias ponderadas en $\mathrm{T} 1 \mathrm{e}$ iso/ hiperintensas en T2. ${ }^{12,13}$

El componente quístico tiene similar señal al LCR en secuencias ponderadas en $\mathrm{T} 1$ y $\mathrm{T} 2$ sin suprimir completamente en FLAIR por su contenido rico en proteínas. $^{12}$

El realce es heterogéneo e irregular con áreas de necrosis asociadas (-Fig. 4). . $^{12,13}$

En la secuencia GRE, pueden apreciarse hipointensidades en las calcificaciones o focos de sangrado y pueden restringir en la secuencia difusión. ${ }^{12,13}$

Algunos ependimomas supratentoriales intraparenquimatosos pueden manifestarse como lesiones expansivas sólido/quísticas con nódulo mural y calcificaciones asociadas. ${ }^{12,13}$
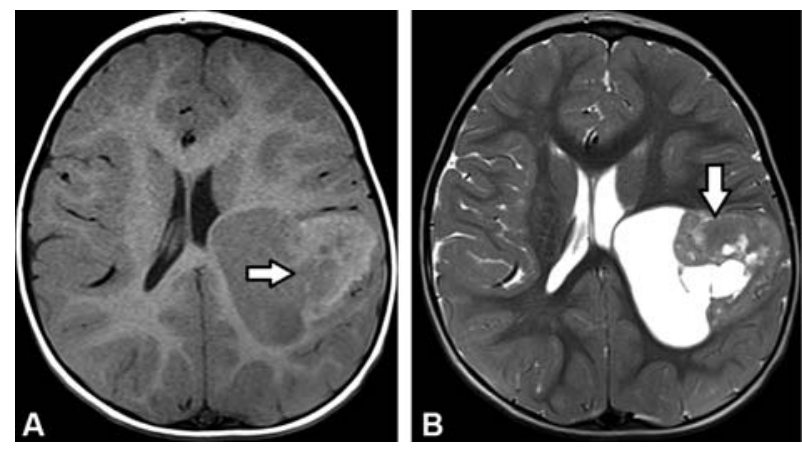

Los ependimomas intraventriculares pueden extenderse al parénquima encefálico adyacente y ocasionar edema vasogénico. Los localizados en el IV ventrículo tienden a rellenarlo como un "molde de yeso" y expandirse por los orificios de Luschka y Magendie originando siembras de secundarismo. ${ }^{12}$

5) Meduloblastoma

Es el tumor maligno más frecuente del sistema nervioso central (SNC) en la edad pediátrica. ${ }^{14,15}$

La clínica depende de la localización, siendo habituales los síntomas por aumento de la presión endocraneal por hidrocefalia obstructiva. ${ }^{14}$

Suelen asociarse con los síndromes de: Turcot tipo 2, Goorlin Gotz, Li Fraumeni, Rubinstein-Taybi, anemia de Fanconi y de Nijmegen. ${ }^{14}$

Frecuentemente se localizan en la fosa posterior de ubicación medial, comprometen al vermis cerebeloso extendiéndose hacia el IV ventrículo y diseminan por vía leptomeníngea, por lo que se aconseja estudiar al neuroeje completamente. $^{14,15}$

En adultos y niños mayores pueden localizarse en los hemisferios cerebelosos y presentar degeneración quística. ${ }^{14,15}$

En TC se aprecian hiperdensos (por hipercelularidad), con variable edema periférico y alrededor del $20 \%$ de los casos pueden presentar calcificaciones. ${ }^{14,15}$

Fig. 4 RM cerebral. Cortes axiales: ponderado en T1 (A), ponderado en T2 (B), DWI (C) ponderado en T1 con gadolinio (D). A nivel parietotemporal izquierdo se objetiva una lesión expansiva sólido-quística, que ejerce efecto de masa contactando con el ventrículo lateral homolateral. Con gadolinio muestra escaso e irregular realce (flecha). Por anatomía patológica se diagnosticó ependimoma. 

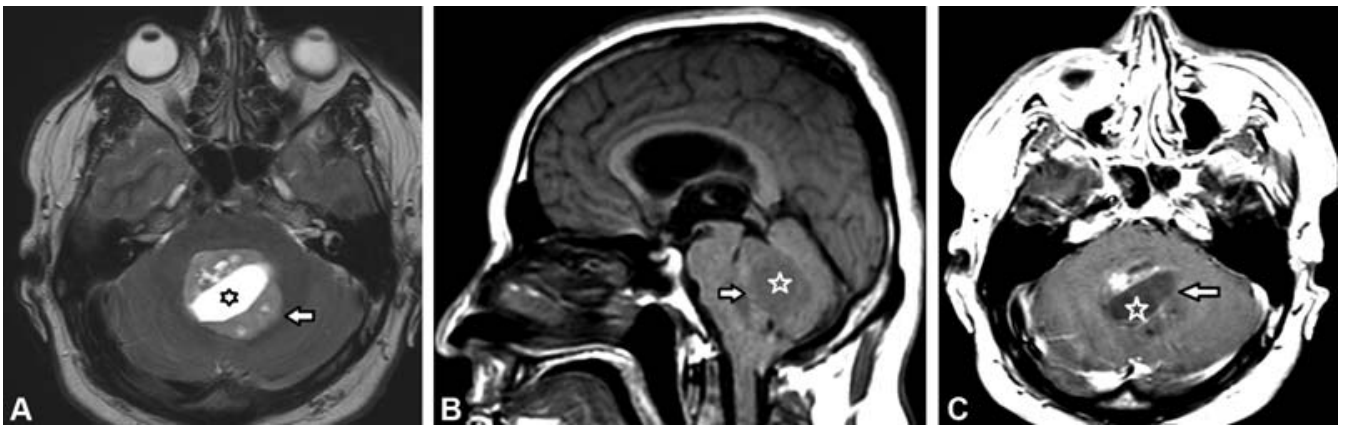

Fig. 5 Meduloblastoma. RM axial ponderada en T2 (A); sagital ponderada en T1 (B) y axial ponderada en T1 con gadolinio (C). Se visualiza una masa expansiva heterogénea, infratentorial, medial, que remodela el IV ventrículo y realza irregularmente con gadolinio (flechas). La secuencia ponderada en $\mathrm{T} 2$ evidencia componente quístico de la misma (estrella).

El realce es variable. ${ }^{14}$

En RM se presentan hipo-isointensos en secuencias ponderadas en $\mathrm{T} 2$, el tumor es predominantemente homogéneo, con poco componente necrótico, hemorrágico o cálcico.

La heterogenicidad en secuencias ponderadas en T2 asociada a realce con patrón "en panal”, detecta la variante anaplásica del meduloblastoma con una sensibilidad del $100 \%$ (-Fig. 5) ${ }^{14,15}$

6) Ganglioglioma

Es un tumor neuroepitelial bien diferenciado que se presenta en niños y jóvenes adultos con predilección del lóbulo temporal, siendo una causa frecuente de epilepsia., 5, 16,17

También puede presentarse en los lóbulos parietal y frontal. ${ }^{16}$

Es una masa sólido-quística que realza, y parcialmente localizada en la periferia de un hemisferio cerebral. ${ }^{16}$

Las calcificaciones son frecuentes y pueden estar rodeadas de edema vasogénico. 5,16

EnTC se observa de aspecto variable, siendo más frecuente la presentación como masas hipodensas con realce irregular/ heterogéneo. ${ }^{16,17}$

En RM tiene una apariencia variable e inespecífica; pueden tener un componente quístico; apreciándose ser hipo/ isointensos en secuencias ponderadas en $\mathrm{T} 1 \mathrm{y}$ asociarse a la displasia cortical y en secuencias ponderadas en $\mathrm{T} 2$ puede ser hiperintenso. ${ }^{5,16,17}$
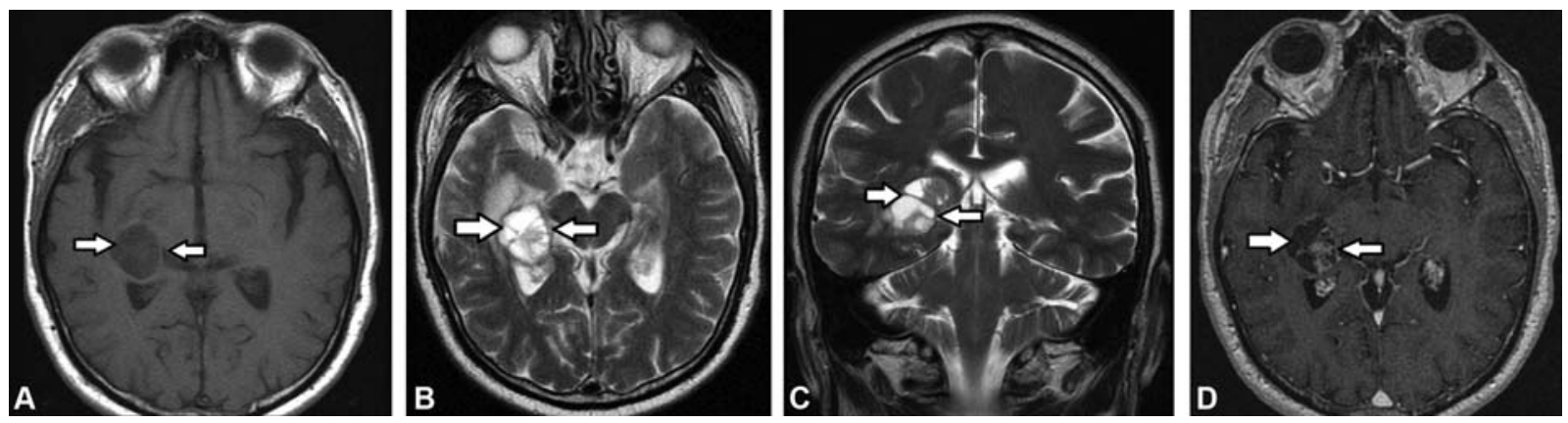

Fig. 6 RM cerebral. Cortes: axial ponderado en T1 (A), axial ponderado en T2 (B), coronal ponderado en T2 (C) y axial ponderado en T1 con gadolinio (D). En el lóbulo temporal derecho, a nivel del hipocampo se objetiva una lesión multiquística septada (flechas), rodeada de edema vasogénico. Comprime parcialmente el asta temporal del ventrículo lateral ipsilateral y realza heterogéneamente con gadolinio. Corresponde a ganglioglioma. 

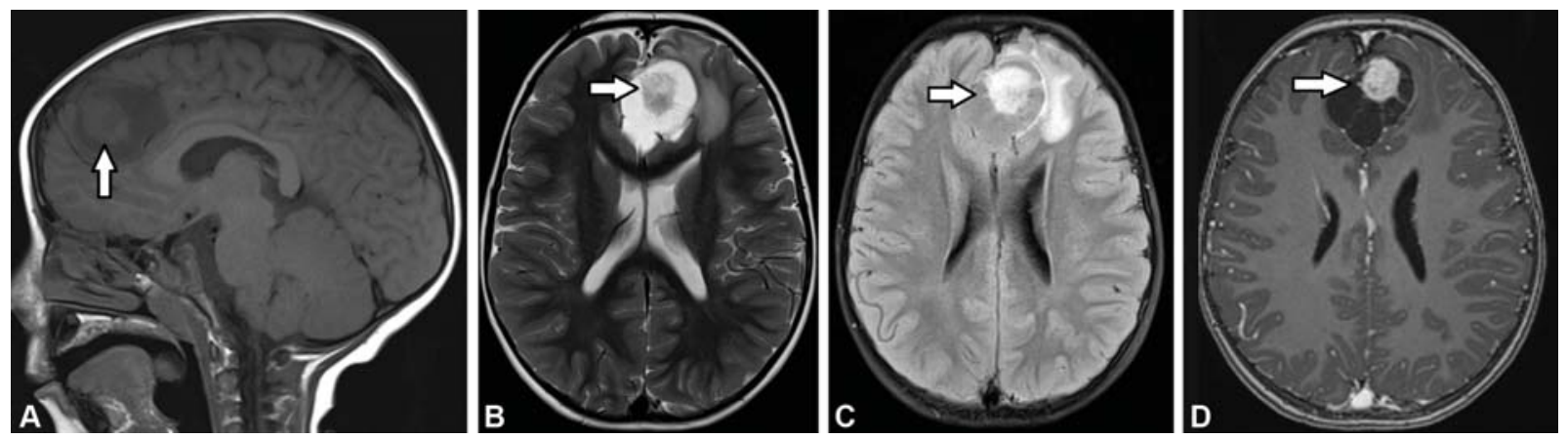

Fig. 7 RM cerebral. Cortes: sagital ponderado en T1 (A), axial ponderado en T2 (B), axial FLAIR (C) y axial ponderado en T1 con gadolinio (D). De localización frontal izquierda, rodeada de edema vasogénico, se pone de manifiesto una lesión expansiva quística que presenta nódulo mural sólido excéntrico con septos finos en el interior (flechas). Tras la administración de gadolinio se objetiva realce intenso del nódulo mural periférico, así como de los septos anteriormente mencionados. Dicha lesión atraviesa la línea media y genera compresión parcial de las prolongaciones frontales de los ventrículos laterales. Por anatomía patológica correspondió a xantoastrocitoma pleomórfico.

Se aprecian en TC como masas hipodensas bien delimitadas. $^{21}$

En RM muestran apariencia "burbujeante" debido a su aspecto sólido/ quístico, siendo esas porciones levemente más hiperintensas que el LCR; mientras que los componentes sólidos se comportan hipointensos en secuencias ponderadas en T1 e hiperintensos en T2. ${ }^{20,21}$

Son cuneiformes, con múltiples septos en su interior, pueden generar remodelación de la tabla interna (60\%) (hallazgo visible en otros tumores corticales) y calcificarse, sin realzar o realzando tenuemente con gadolinio (-Fig. 8). ${ }^{19-21}$

Se caracterizan por presentar el "signo del anillo hiperintenso en FLAIR": constituye un fino anillo hiperintenso en FLAIR, bien delimitado en la periferia, separando el tumor del resto del parénquima cerebral sano. ${ }^{19}$

9) Glioblastoma (ex glioblastoma multiforme)

La Organización Mundial de la Salud (OMS) realizó una actualización en 2016 (-Tabla 3), modificando la antigua denominación del glioblastoma multiforme en glioblastoma con gen IDH salvaje (no mutado), con gen IDH mutado y glioblastoma NOS (no especifica si hay o no mutación). ${ }^{22}$
El glioblastoma es el tumor maligno primitivo más frecuente en el cerebro de los adultos, representando entre el 12\%-15\% de todas las neoplasias intracraneales. ${ }^{23,24}$

Predominan en hombres (3:2) entre los 45-70 años de edad, siendo los hemisferios cerebrales su localización más frecuente. La ubicación infratentorial o espinal es rara. 24,25

El pronóstico suele ser desfavorable por su alto grado de malignidad, con una sobrevida variable, dependiendo del tipo (secundario o de novo), estimada en 14 meses. ${ }^{25,26}$

Puede presentarse de nuevo ( $>90 \%$ ) o progresar de un tumor existente de bajo grado $(<10 \%){ }^{24,27}$

En RM se presentan como una lesión expansiva heterogénea en los hemisferios cerebrales, de bordes mal delimitados, tienen sectores de apariencia quística por abundantes áreas de necrosis y hemorragia, realzan heterogéneamente con gadolinio y están rodeados de importante edema peritumoral (-Fig. 9). ${ }^{24,25}$

Suelen infiltrar el cuerpo calloso atravesando la línea media a través de él, comprometiendo el hemisferio cerebral contralateral (glioma en "alas de mariposa"). ${ }^{24}$
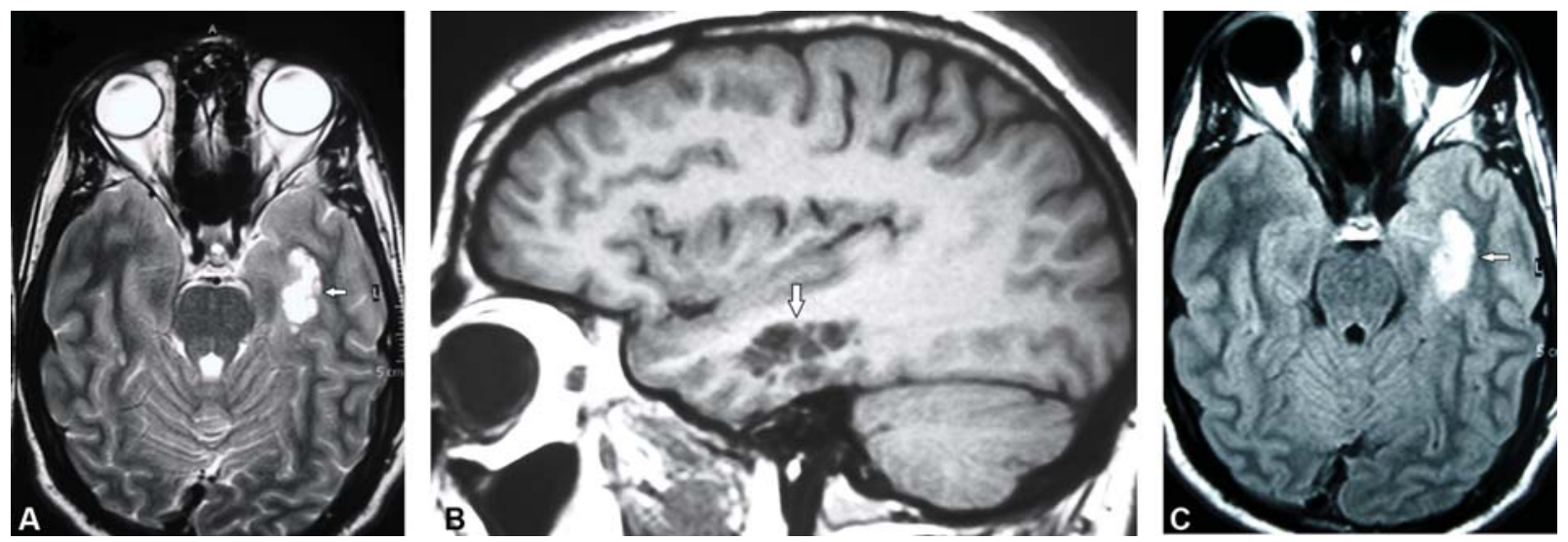

Fig. 8 RM de cerebro. Cortes: axial ponderado en T2 (A), sagital ponderado en T1 (B) y axial FLAIR (C). En el lóbulo temporal izquierdo se evidencia una lesión microquística multinodular sin edema periférico ni generar efecto de masa (flechas). La secuencia FLAIR muestra tenue anillo hiperintenso periférico. Correspondió a DNET. 
Tabla 3 Clasificación de la OMS 2016 adaptada a los tumores de apariencia quística

\begin{tabular}{|c|c|c|}
\hline Tumor & Marcador molecular & Grado tumoral \\
\hline Astrocitoma pilocítico & BRAF alterado & 1 \\
\hline Hemangioblastoma & - & I \\
\hline Neurocitoma central & - & II \\
\hline Ependimoma & RELA fusión positiva & II/III \\
\hline Meduloblastoma & $\begin{array}{l}\text { WNT activado } \\
\text { SHH activado (TP53 mutado) } \\
\text { SHH activado (TP53 salvaje) } \\
\text { Grupo } 3 \\
\text { Grupo } 4\end{array}$ & $\begin{array}{l}\text { IV } \\
\text { (todos los subtipos) }\end{array}$ \\
\hline Ganglioglioma & - & I \\
\hline $\begin{array}{l}\text { Xantoastrocitoma } \\
\text { Pleomórfico }\end{array}$ & BRAF alterado & II \\
\hline DNET & - & I \\
\hline Glioblastoma & $\begin{array}{l}\text { IDH salvaje } \\
\text { IDH mutado } \\
\text { NOS }\end{array}$ & $\begin{array}{l}\text { IV } \\
\text { (todos los subtipos) }\end{array}$ \\
\hline Menigioma & - & I \\
\hline Schwanomma & - & I \\
\hline Craneofaringioma & - & I \\
\hline
\end{tabular}

Fuente: Referencia bibliográfica 22.

Muestran sectores con restricción en difusión debido a la alta celularidad y en la tractografía por RM demuestran destrucción o infiltración de fibras y haces. ${ }^{24,27}$

La espectroscopía puede proporcionar información valiosa para caracterizar a la lesión como del parénquima circundante. Hay elevación de Colina por abundante proliferación de membrana celular, marcada disminución del $\mathrm{N}$ Acetilaspartato por muerte neuronal así como elevación del pico de lactato/lípidos vinculable al componente quísticonecrótico, sugiriendo una lesión neoplásica. ${ }^{24}$

En la perfusión por RM, la elevación del volumen sanguíneo cerebral relativo (VSCr o rCBV) constituye un marcador de neovascularización en una lesión glial de alto grado. ${ }^{24,27}$

10) Metástasis
La presencia de una lesión encefálica en el contexto de un paciente con tumor conocido sugiere la posibilidad de una metástasis. ${ }^{28}$

El origen de esos tumores suele ser: pulmón, mama, melanoma, riñón y tumores del tracto digestivo. ${ }^{28}$

Generalmente, asientan en la unión sustancia gris/blanca de los hemisferios cerebrales, siguiendo el territorio de irrigación de las arterias cerebrales. ${ }^{29}$

En TC se aprecian iso/hipodensas rodeadas de edema vasogénico, pudiendo ser omitidas si no se administra contraste e.v. ${ }^{23}$

En RM las lesiones se aprecian generalmente hipointensas en secuencias ponderadas en T1 e hiperintensas en T2, pueden demostrar restricción en difusión y realzar en
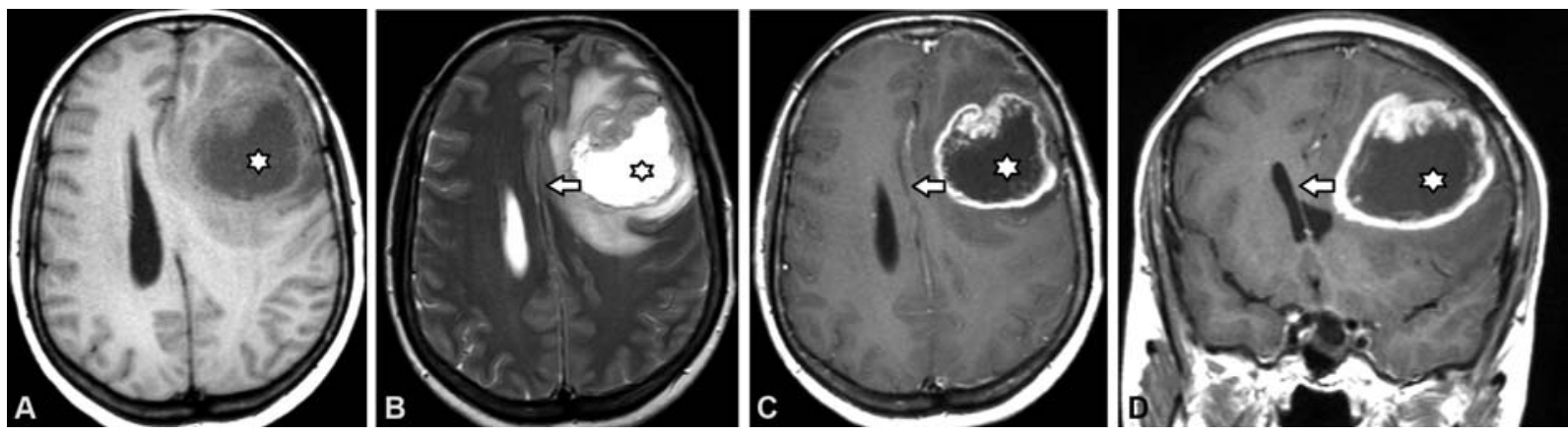

Fig. 9 GBM. RM de cerebro. Cortes: axial ponderado en T1 (A), ponderado en T2 (B) y axial (C) y coronal ponderado en T1 con gadolinio (D). En el lóbulo frontal izquierdo se evidencia una masa expansiva sólido-quística (estrella) con áreas necróticas asociadas. Se acompaña de edema vasogénico provocando efecto de masa, desplazando la línea media hacia la derecha (flechas) y comprimiendo a la prolongación frontal del ventrículo lateral ipsilateral. 

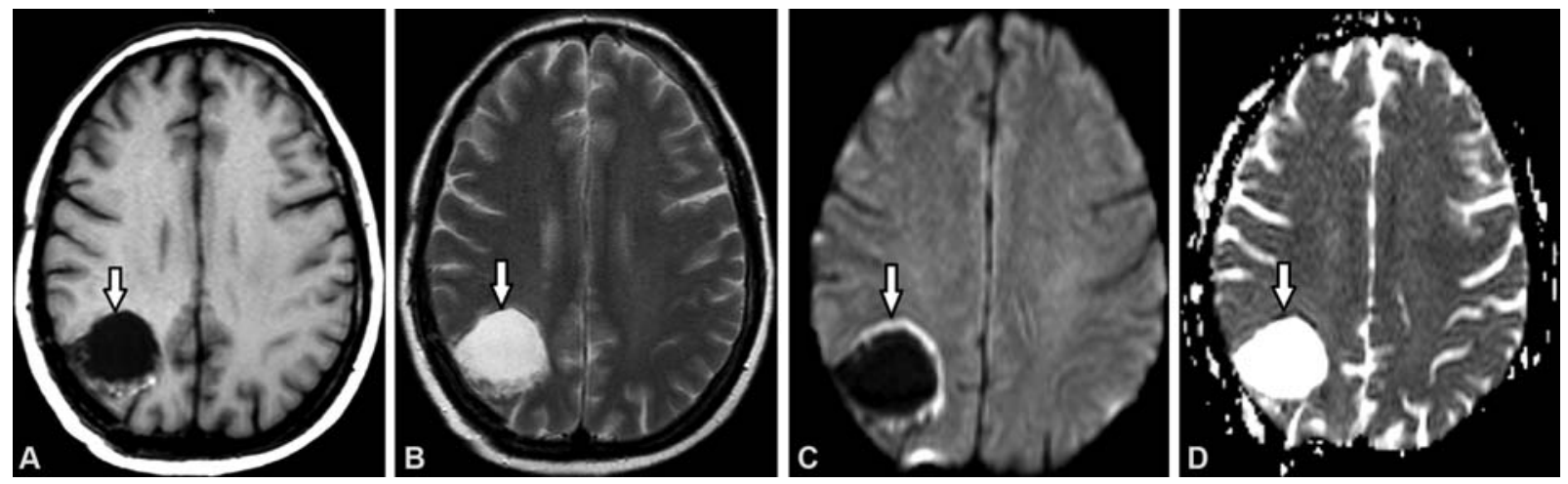

Fig. 10 Metástasis quística de cáncer de pulmón. RM de cerebro. Cortes: axial ponderado en T1 (A), ponderado en T2 (B), DWI (C) y mapa de ADC (D). En el lóbulo parietal derecho se observa una lesión quística (flechas) con engrosamiento focal de su pared en la región posterior, presentando restricción periférica incompleta en la secuencia de difusión. Por anatomía patológica se confirmó secundarismo de su patología de base.

forma anular, puntiforme o nodular (en forma completa 0 irregular) con gadolinio. ${ }^{28-30}$

$\mathrm{Si}$ son grandes o muy agresivas pueden tener necrosis central y/o cavitaciones ( $\mathbf{- F i g . ~ 1 0 ) . 2 8 , 2 9}$

\section{Tumores extra-axiales}

Algunos tumores extra-axiales pueden sufrir degeneración quística, o bien asociarse a imágenes quísticas no neoplásicas. Son ejemplos de ellos: meningiomas, schwannomas, craneofaringiomas o macroadenomas hipofisarios (-Figs. 11-13). ${ }^{31}$

El 10-20\% de los meningiomas pueden tener degeneración quística, mostrando densidad o señal similar al LCR. ${ }^{4,31}$
Las calcificaciones se valoran mejor en TC. ${ }^{4}$

Los quistes peritumorales que ocurren próximos a los schwannomas pueden ser quistes aracnoideos; los meningiomas pueden atrapar LCR entre el tumor y la corteza cerebral. ${ }^{31}$

Los craneofaringiomas o los macroadenomas hipofisarios con extensión supraselar pueden obstruir y dilatar espacios perivasculares. ${ }^{31,32}$

Los quistes peritumorales así como los espacios perivasculares dilatados no realzan, pudiéndolos diferenciar de los tumores. ${ }^{31}$

La correcta descripción y caracterización de una imagen de apariencia quística en el SNC permite una aproximación diagnóstica más específica de la misma.
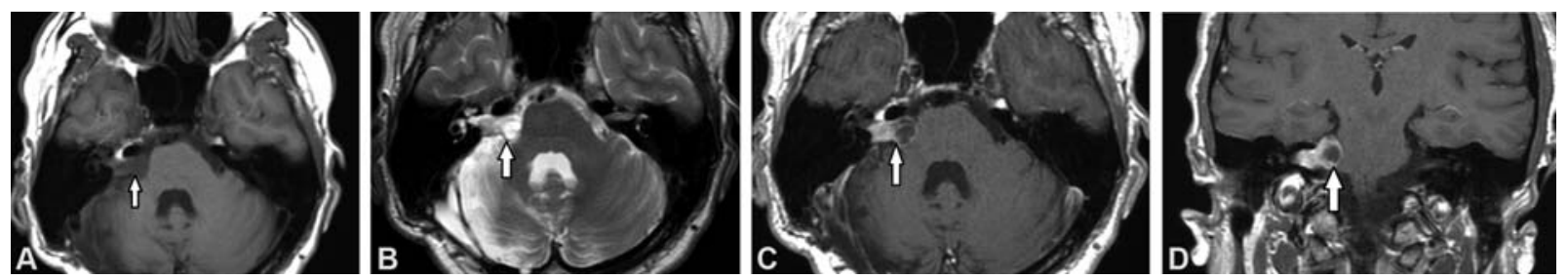

Fig. 11 RM de peñascos. Cortes: axial ponderado en T1 (A), axial ponderado en T2 (B), axial (C) y coronal (D) ponderados en T1 con gadolinio. En el ángulo pontocerebeloso derecho se pone de manifiesto (flechas) una lesión heterogénea, con componente quístico que contacta en su borde medial con la protuberancia y en su borde lateral ensancha el porus acústico, ingresando en el CAl hasta contactar con el fundus. Realza en forma irregular tras la administración de gadolinio. Corresponde a neurinoma del VIII par con componente quístico.
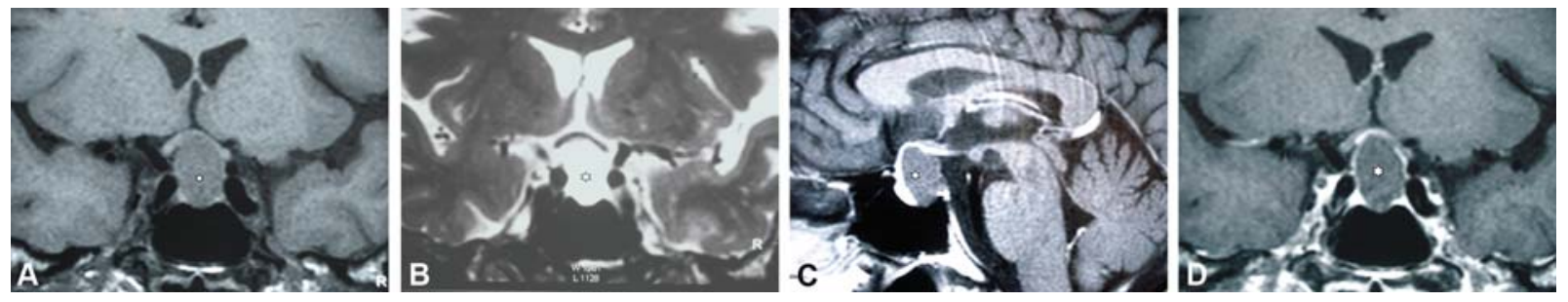

Fig. 12 RM de hipófisis. Corte coronal ponderado en T1 (A), coronal ponderado en T2 (B), sagital ponderado en T1 con gadolinio (C) y coronal ponderado en T1 con gadolinio (D). Alteración de la morfología de la glándula hipofisaria a expensas de una lesión de apariencia quística (estrella), que no realza tras la administración de gadolinio. La misma se extiende cefálicamente hasta contactar y desplaza al quiasma óptico. Corresponde a un macroadenoma quístico. 

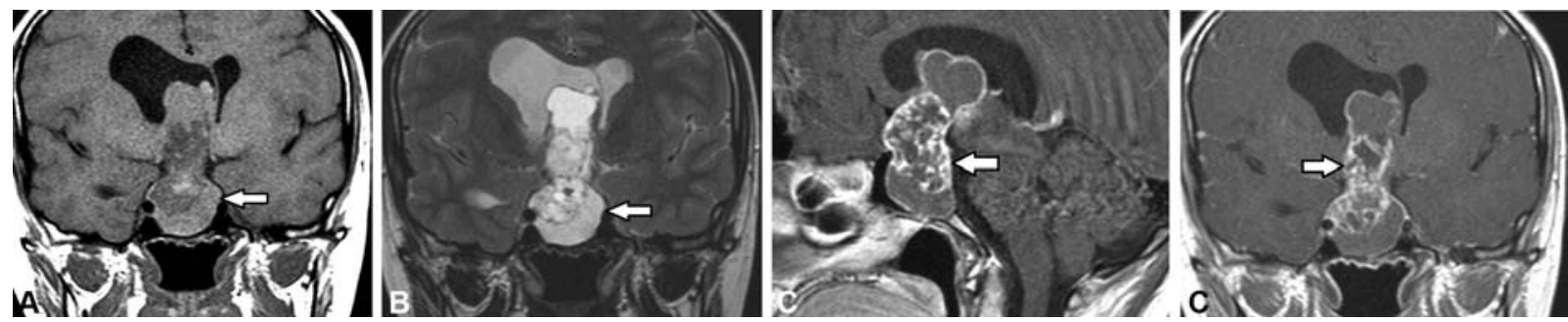

Fig. 13 RM de región selar. Cortes: coronal ponderado en T1 (A), coronal ponderado en T2 (B) y sagital (C) y coronal ponderado en T1 (D) con gadolinio. Se aprecia (flecha) alteración de la morfología selar a expensas de una lesión expansiva sólido-quística con áreas espontáneamente hiperintensas en secuencia ponderada en T1 por contenido rico en proteínas/sangre, asociado a contenido heterogéneo. Presenta realce irregular luego de la administración de gadolinio. Lateralmente invade a los senos cavernosos y caudalmente al seno esfenoidal. Cefálicamente se extiende hasta contactar y desplazar al quiasma óptico invadiendo al III ventrículo y ventrículos laterales. Corresponde a craneofaringioma.

Eso deriva en una reducción del tiempo diagnóstico, facilitando una terapéutica específica para cada paciente (-Tabla 1).

\section{Responsabilidades Éticas}

Protección de personas y animales. Los autores declaran que para esta investigación no se han realizado experimentos en seres humanos ni en animales.

Confidencialidad de los datos. Los autores declaran que han seguido los protocolos de su centro de trabajo sobre la publicación de datos de pacientes.

Derecho a la privacidad y consentimiento informado. Los autores declaran que en este artículo no aparecen datos de pacientes.

\section{Conflicto de Intereses}

Pablo Sartori declara como posible conflicto de interés ser miembro del Capítulo de Neuroradiología de la Sociedad Argentina de Radiología (SAR).

Nicolás Sgarbi declara como posible conflicto de interés ser presidente de la Sociedad de Radiología e Imagenología del Uruguay (SRIU).

\section{Bibliografía}

1 Sartori P, Sgarbi N. Tomografía computada y resonancia magnética de variantes normales/congénitas de apariencia quística y presentación frecuente en el encéfalo. Rev Argent Radiol. 2019;83(01):12-22

2 Bell D, Gaillard F, et al. Cyst. Radiopaedia Web site. https:// radiopaedia.org/articles/cyst?lang=us. Accesed June 20, 2020

3 Cueva Sánchez MA, Morales Barrera ME, Ramos Garibay A. Quiste epidérmico de localización poco frecuente. Reporte de un caso. Rev Cent Dermatol Pascua. 2001;10(02):100-102

4 Alandete SPG, Meseguer MA, De la Via E, Uceda D, Poyatos C. Differential diagnosis of intracranial cystic lesions. Epos Web site. https://epos.myesr.org/esr/viewing/index.php? module=viewing_poster\&task=\&pi=126511. Accesed June 21, 2020.

5 Raz E, Zagzag D, Saba L, Mannelli L, Di Paolo PL, Ambrosio FD, et al. Cyst with a mural nodule tumor of the brain. Cancer Imaging. 2012;12(01): 237-244
6 Chourmouzi D, Papadopoulou E, Konstantinidis M, Syrris V, Kouskouras K, Haritanti A, et al. Manifestations of pilocytic astrocytoma: a pictorial review. Insights Imaging. 2014;5(03):387-402

7 Docampo J, González N, Muñoz A, Bruno C, Morales C. Astrocitoma pilocítico. Formas de presentación. Rev Argent Radiol. 2014;78(02):68-81

8 Poretti A, Meoded A, Huisman TAGM. Neuroimaging of pediatric posterior fossa tumors including review of the literature. J Magn Reson Imaging. 2012;35(01):32-47

9 Shin JH, Lee HK, Khang SK, Kim DW, Jeong AK, Ahn KJ, et al. Neuronal tumors of the central nervous system: radiologic findings and pathologic correlation. Radiographics. 2002;22 (05):1177-1189

10 Smoker WRK, Townsend JJ, Reichman MV. Neurocytoma accompanied by intraventricular hemorrhage: case report and literature review. AJNR Am J Neuroradiol. 1991;12(04):765-770

11 Conrad MD, Morel C, Guyotat J, Pelissou-Guyotat I, Saint-Pierre G, Deruty R. Neurocitomas do sistema nervoso central: análise clínico-patológica de três casos. Arq Neuropsiquiatr. 2000;58 (04):1100-1106

12 Smith AB, Smirniotopoulos JG, Horkanyne-Szakaly I. From the radiologic pathology archives: intraventricular neoplasms: radiologic-pathologic correlation. Radiographics. 2013;33(01):21-43

13 Mermuys K, Jeuris W, Vanhoenacker PK, Van Hoe L, D’Haenens P. Best cases from the AFIP: supratentorial ependymoma. Radiographics. 2005;25(02):486-490

14 Martínez León MI. Meduloblastoma pediátrico, revisión y puesta al día. Radiologia (Madr). 2011;53(02):134-145

15 Raleigh DR, Varenika V, Tihan T, Haas Kogan DA. Clinical, Histopathologic, Radiographic and Molecular Classification of Medulloblastoma. JSM Clin Oncol Res. 2014;2(04):10-25

16 Koeller KK, Henry JMArmed Forces Institute of Pathology. From the archives of the AFIP: superficial gliomas: radiologicpathologic correlation. Radiographics. 2001;21(06):1533-1556

17 Zentner J, Wolf HK, Ostertun B, Hufnagel A, Campos MG, Solymosi L, et al. Gangliogliomas: clinical, radiological, and histopathological findings in 51 patients. J Neurol Neurosurg Psychiatry. 1994;57(12):1497-1502

18 Gonçalves VT, Reis F, Queiroz LdeS, França M Jr. Pleomorphic xanthoastrocytoma: magnetic resonance imaging findings in a series of cases with histopathological confirmation. Arq Neuropsiquiatr. 2013;71(01):35-39

19 Raz E, Kapilamoorthy TR, Gupta AK, Fiorelli M. Case 186: Dysembrioplastic neuroepithelial tumor. Radiology. 2012;265 (01):317-320

20 Reyes Botero G, Millán Giraldo PA, Usuga Arcilla H, Uribe Uribe CS, Góez Gutiérrez EI. Tumor disembrioplástico neuroepitelial y epilepsia focal de larga evolución: informe de caso y revisión en la literatura. Acta Neurol Colomb. 2008;24(01):33-37 
21 Patel K, Rathod G. Dysembryoplastic neuroepithelial tumor: A rare case report. IAIM. 2015;2(05):164-167

22 Louis D, Perry A, Reifenberg G, von Deimling A, Figarella BrangerD, Cavenee W, et al. The 2016 World Health Organization Classification of Tumors of the Central Nervous System: a summary. Acta Neuropathol. 2016;131(06):803-820

23 Gevaert O, Mitchell LA, Achrol AS, Xu J, Echegaray S, Steinberg GK, et al. Glioblastoma multiforme: exploratory radiogenomic analysis by using quantitative image features. Radiology. 2014; 273(01):168-174

24 Altman DA, Atkinson DS Jr, Brat DJ. Best cases from the AFIP: glioblastoma multiforme. Radiographics. 2007;27(03):883-888

25 Aljure VJ, Pulido-Arias EA, Rodríguez-Monroy JA, Rodríguez-Mateus MN, Ramos-Hernández JA. Diagnóstico Diferencial de Lesiones Cerebrales con Realce en Anillo en Tomografía Computada y Resonancia Magnética. Duazary. 2016;13(02):149-158

26 Zhou J, Reddy M, Wilson B, Blair D, Taha A, Frampton C, et al. MR Imaging Characteristics Associate with Tumor-Associated Macrophages in Glioblastoma and Provide an Improved
Signature for Survival Prognostication. AJNR Am J Neuroradiol. 2018;39(02):252-259

27 Kao HW, Chiang SW, Chung HW, Tsai FY, Chen CY. Advanced MR imaging of gliomas: an update. BioMed Res Int. 2013; 2013:970586

28 Oprişan A, Popescu BO. Intracranial cysts: an imagery diagnostic challenge. ScientificWorldJournal. 2013;2013; 172154

29 Sharma V, Prabhash K, Noronha V, Tandon N, Joshi A. A systematic approach to diagnosis of cystic brain lesions. South Asian J Cancer. 2013;2(02):98-101

30 Lignelli A, Khandji AG. Review of imaging techniques in the diagnosis and management of brain metastases. Neurosurg Clin N Am. 2011;22(01):15-25, v

31 Osborn AG, Preece MT. Intracranial cysts: radiologic-pathologic correlation and imaging approach. Radiology. 2006;239(03): 650-664

32 Curran JG, O'Connor E. Imaging of craniopharyngioma. Childs Nerv Syst. 2005;21(8-9):635-639 